\title{
Low Voltage, High-Q SOI MEMS Varactors for RF Applications
}

\author{
Yalcinkaya, Arda Deniz; Jensen, Søren; Hansen, Ole
}

Published in:

Proceedings of the 29th EuropeanSolid-State Circuits Conference (ESSCIRC)

Link to article, DOI:

10.1109/ESSCIRC.2003.1257208

Publication date:

2003

Document Version

Publisher's PDF, also known as Version of record

Link back to DTU Orbit

Citation $(A P A)$ :

Yalcinkaya, A. D., Jensen, S., \& Hansen, O. (2003). Low Voltage, High-Q SOI MEMS Varactors for RF Applications. In Proceedings of the 29th EuropeanSolid-State Circuits Conference (ESSCIRC) IEEE. https://doi.org/10.1109/ESSCIRC.2003.1257208

\section{General rights}

Copyright and moral rights for the publications made accessible in the public portal are retained by the authors and/or other copyright owners and it is a condition of accessing publications that users recognise and abide by the legal requirements associated with these rights.

- Users may download and print one copy of any publication from the public portal for the purpose of private study or research.

- You may not further distribute the material or use it for any profit-making activity or commercial gain

- You may freely distribute the URL identifying the publication in the public portal 


\title{
Low Voltage, High-Q SOI MEMS Varactors for RF Applications
}

\author{
Arda D. Yalçınkaya, Søren Jensen and Ole Hansen \\ Mikroelektronik Centret (MIC), Ørsteds Plads B-345-East DTU, \\ Technical University of Denmark, DK-2800, Kgs. Lyngby, Denmark \\ e-mail:ady@mic.dtu.dk
}

\begin{abstract}
A micro electromechanical tunable capacitor with a low control voltage, a wide tuning range and high electrical quality factor is presented with detailed characterizations. A $50 \mu \mathrm{m}$ thick single-crystalline silicon layer was etched using deep reactive ion etching (DRIE) for obtaining high-aspect ratio (> 20) parallel comb-drive structures with vertical sidewalls. Consisting of only one lithographic mask step, the process sequence can be completed in a short time. Having $I p F$ of nominal capacitance, the device offers an electrical quality factor of 100 at $100 \mathrm{MHz}$ and a self resonance at $4.08 \mathrm{GHz}$, enabling the usage as a passive component in the RF band. The mechanical settling time of the varactor is measured to be $200 \mu \mathrm{s}$ in ambient air. For control voltages ranging from $1.5 \mathrm{~V}$ to $5 \mathrm{~V}$, tuning ratios of $2: 1,1.7: 1$ and 1.5:1 are obtained from different capacitor designs. It was found that the device is a suitable passive component to be used in band-pass filtering, voltage controlled oscillator or impedance matching applications in the Very High Frequency (VHF) and Ultra High Frequency (UHF) bands.
\end{abstract}

\section{Introduction}

Among the applications of tunable capacitors are voltage controlled oscillators, tunable filters and resonators. High quality factor band pass filter of Intermediate Frequency (IF) blocks in heterodyne receivers (with center frequencies ranging from $455 \mathrm{kHz}$ to $254 \mathrm{MHz}$ ) is an example where high quality factor varactors can be employed. Additionally, a tunable capacitor with a wide tuning range is a useful component for impedance matching purposes, as in the case of matching between the active interface circuitry (e.g. low-noise amplifier) and the passive components (e.g. $50 \Omega$ antenna) for maximum power transfer. Conventionally, RF blocks use electronic varactors implemented through diodes or transistors. Advances in micro electromechanical system technologies have allowed implementation of MEMS varactors, as an alternative technology to be used in the aforementioned applications.

The electrical quality factor of surface micromachined devices is limited by the spring which has to be compliant to enable low-voltage electrostatic actuation $[1,2,3,5]$.
Since a compliant spring can be realized using narrow and long flexural beams, the main contribution to the serial resistance of the capacitor is defined by them. In order to reduce the series resistance (thus to increase the quality factor), normally, either metallic structural layers or deposition of thick metals on the RF signal path have to be used.

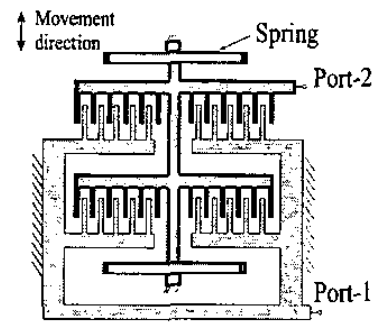

(a)

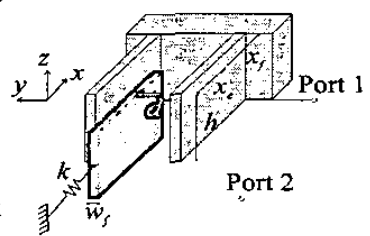

(b)
Figure 1. (a) Tunable capacitor schematic (b) Definitions for the geometries for comb fingers.

\section{Design considerations}

Figure 1(a) shows the mechanical schematic of the interdigitated comb drive MEMS tunable capacitor. A movable part (the shuttle), marked as port-1, is suspended by a couple of folded beam springs with a low effective spring constant, $k$, for motion in the intended direction the $x$-direction - and a high spring constant for motion in other directions. In Figure 1(b), a detailed sketch of one comb finger-pair with a schematic spring is given (refer to Table 1 for the definitions of the dimensions). Due to the symmetry in the system, applied bias voltages do not change the air gap $(d)$. Ignoring fringe fields, $N$ identical finger-pairs contribute an intended capacitance, $C_{I}$, of

$$
C_{I}(x)=2 N \varepsilon_{0} \frac{h}{d}\left(x_{e}+x\right)=C_{I 0}\left(1+\frac{x}{x_{e}}\right)
$$

where $x$ is the displacement of the shuttle. Inherent to the design, however, is also an unwanted parasitic capacitance, $C_{P}$, between the end of the fingers and the finger connector bar, this capacitance limits the stroke length of the tunable capacitor. To a first approximation the parasitic capacitance for $N$ fingers-pairs is obtained from 


$$
C_{P}(x)=2 N \varepsilon_{0} \frac{h w_{\mathrm{f}}}{x_{\mathrm{f}}-x}=\frac{C_{P 0}}{1-\frac{x}{x_{\mathrm{f}}}} .
$$

The displacement, $x$, of the capacitor with an applied bias voltage is obtained by examining the potential energy, $\mathcal{U}$, of the system including the voltage source. The displacement is the solution to $\frac{\partial u}{\partial x}=0$, provided $\frac{\partial^{2} u}{\partial x^{2}}>0$, otherwise the system is meta-stable and the fingers will snap to the finger connector bars [4]. The total change in potential energy of the system is

$$
\mathcal{U}=\frac{1}{2} k x^{2}-\frac{1}{2} V^{2}\left(C_{I}+C_{P}\right) .
$$

At small displacements for a well designed device with $d w_{\mathrm{f}} \ll x_{\mathrm{f}}^{2}$, the displacement is approximately $x \simeq$ $\frac{1}{2} V^{2} 2 N \varepsilon_{0} \frac{h}{d} \frac{1}{k}$ and therefore the expected capacitance as a function of the applied bias is

$$
C_{I}=C_{I 0}\left(1+V^{2} \frac{N \varepsilon_{0} h}{k x_{e} d}\right)=C_{I 0}\left(1+V^{2} \frac{C_{I 0}}{2 k x_{e}^{2}}\right) .
$$

The stability requirement $\frac{\partial^{2} u}{\partial x^{2}}>0$, restricts the maximum stable stroke (or travel) length $\left(x_{\max }\right)$;

$\frac{x_{\max }}{x_{\mathrm{f}}}=1-\alpha\left(\sqrt{\alpha^{3}+\alpha^{2}}-\alpha\right)^{-\frac{1}{3}}+\left(\sqrt{\alpha^{3}+\alpha^{2}}-\alpha\right)^{\frac{1}{3}}$

where $\alpha=\frac{d w_{F}}{x_{s}^{2}}$. At very small values of $\alpha$, the maximum relative stroke length is close to 1 , whereas at very high values of $\alpha$, the maximum relative stroke length is limited to $1 / 3$, as found in parallel plate actuators. The maximum stroke length limits the capacitor tuning range, since

$$
\Delta C_{I \max }=C_{I}\left(x_{\max }\right)-C_{I 0}=C_{I 0} \frac{x_{\max }}{x_{\mathrm{f}}} \frac{x_{\mathrm{f}}}{x_{e}} .
$$

The intended and the measured geometries/parameters of the device are summarized in Table 1. Acceleration sensitivity of the device is defined as

$$
S_{m}=\frac{m}{k}=\frac{1}{\omega_{0}^{2}}
$$

where $m$ and $\omega_{0}$ are the effective mass and the mechanical resonance frequency of the device. In order to reduce the $S_{m}$ to avoid unwanted oscillations and displacements due to parasitic mechanical excitations, a hollow spine is incorporated in the design which reduces the effective mass (see Figure 2). Moreover, having the finger length defined by equation 4 , the finger width is designated to be narrow $\left(\frac{x_{e}+x_{f}}{w_{f}} \gg 1\right)$ to minimize the contribution to the effective mass. However, a lower limit to finger width is put by the snap-in phenomenon, where due to lithography and etch variations in the fabrication, one finger experiences an unbalanced electrostatic force causing a snapping to the neighboring finger. Therefore, for a given maximum bias voltage, the fingers have to be stiff enough in the $y$-direction to account for the process variations. This poses a trade-off between the mechanical sensitivity and the snap-in instability. Additionally, narrow air gaps and thick fingers helps to obtain a heavily damped mechanical response, ensuring a fast recovery in the time domain.
Table 1. Designed and fabricated geometries/parameters of the comb-driven tunable capacitor.

\begin{tabular}{lcc}
\hline Parameter & Designed & Fabricated \\
\hline Spring width, $w(\mu \mathrm{m})$ & 1 & 0.8 \\
Spring length, $L(\mu \mathrm{m})$ & 500 & 500 \\
Capacitive gap, $d(\mu \mathrm{m})$ & 2 & 2.7 \\
Device height, $h(\mu \mathrm{m})$ & 50 & 50 \\
Finger width, $w_{\mathrm{f}}(\mu \mathrm{m})$ & 4 & 3.5 \\
Travel path length, $x_{\mathrm{f}}(\mu \mathrm{m})$ & 30 & 30 \\
Comb engagement, $x_{e}(\mu \mathrm{m})$ & 10 & 9 \\
Number of fingers, $N$ & 336 & 336 \\
Device size $(\mu \mathrm{m} \times \mu \mathrm{m})$ & $1100 \times 700$ & $1100 \times 700$ \\
Static capacitance, $C_{I 0}(\mathrm{pF})$ & 1.0 & 0.9 \\
\hline
\end{tabular}

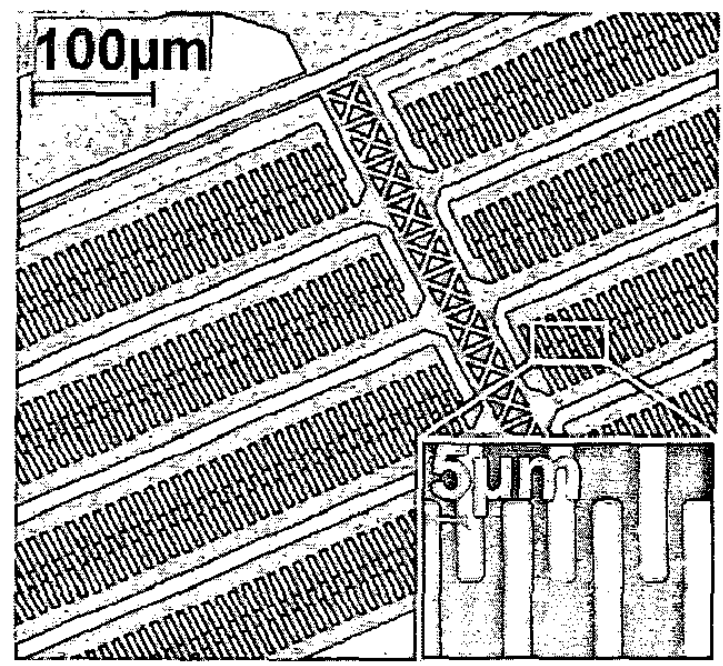

Figure 2. Overview SEM picture of the tunable capacitor. Inset: the detail of the comb-fingers.

\section{Fabrication}

The tunable capacitors are fabricated on an silicon-oninsulator (SOI) substrate in a simple single-mask process using dry deep reactive ion etching (DRIE) followed by a wet isotropic release etch of a sacrificial silicon dioxide layer. The starting material was a 4" (100) SOI substrate with a $50 \mu \mathrm{m}$ thick, Sb doped device layer on top of a $1 \mu \mathrm{m}$ thick oxide layer, and a $465 \mu \mathrm{m}$ thick handle layer. The process sequence, shown in Figure 3, can be summarized as follows.

First, the geometry of the structures was patterned onto the substrate with $1.5 \mu \mathrm{m}$ thick AZ5214 photoresist (Figure 3-A). The masked substrate was then etched anisotropically in the DRIE tool until the buried oxide was reached. An air gap aspect ratio (d:h in Figure 1) of more than 1:20 was obtained, yielding a high capacitance between the fingers (Figure 3-B). After the DRIE step, the remaining photoresist was stripped in an $\mathrm{O}_{2} / \mathrm{N}_{2}$ plasma, and the 


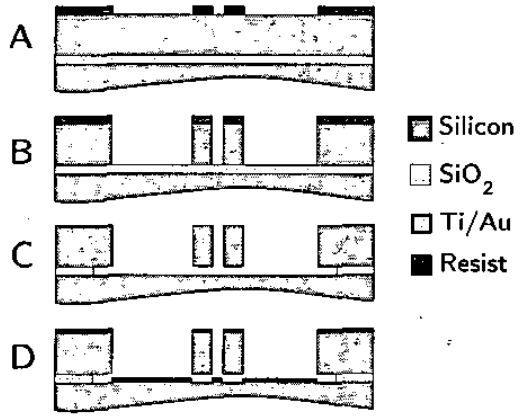

Figure 3. Tunable capacitor fabrication sequence. The silicon is etched using deep reactive ion etching (B). Afterwards, the narrow structure in the middle is released during the etch of the sacrificial silicon dioxide layer while the larger structures on the sides remain anchored (C). Finally, a $\mathrm{Ti} / \mathrm{Au}$ layer is deposited on top of the device (D).

narrow structures released in $40 \%$ hydrofluoric acid (Figure 3-C). Finally, a layer of $200 \AA$ titanium and $5800 \AA$ gold was evaporated on top of the devices to enhance the electrical quality factor (Figure 3-D).

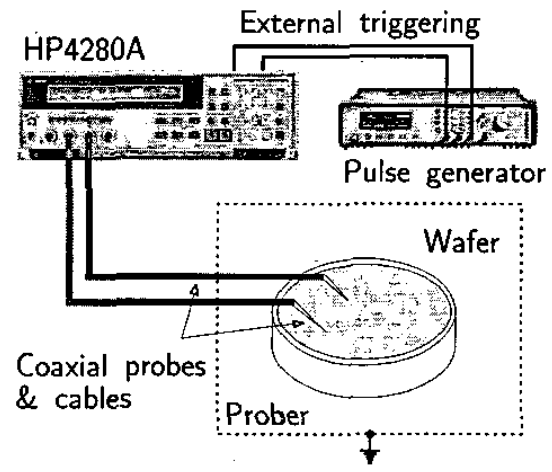

Figure 4. Characterization setup used for extracting $C-V$ and $\mathrm{C}-\mathrm{t}$ curves of the tunable capacitor. The measurements are performed at $1 \mathrm{MHz}$.

\section{Device characterization}

The tunable capacitors were characterized in air to extract the capacitance tuning curve and dynamic behavior. The measurement setup, which allows wafer-level characterization, is shown in Figure 4. Open circuit calibration was performed before the measurements in order to compensate for the probe and pad capacitances [2]. The measured net device capacitance at $1 \mathrm{MHz}$ is shown in Figure 5, revealing that the capacitance can be tuned between $1 \mathrm{pF}$ (static capacitance) and $2 \mathrm{pF}$ by increasing the control voltage to $3.15 \mathrm{~V}$ where maximum deflection is obtained. Furthermore, in Figure 5, the expected capacitance from equation 4 using fabricated dimensions is overlayed on the experimental data, and is in good agree- ment with measurements. The experimental tuning range is $100 \%$, mainly limited by the maximum excursion allowed by the spring anchors.

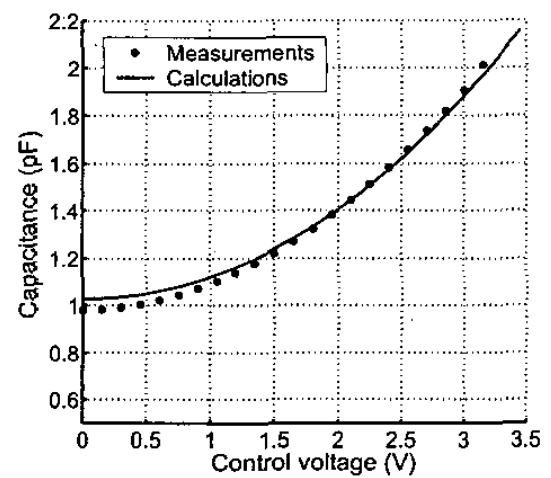

Figure 5. Capacitance-voltage tuning characteristic of the device shown in Figure 2. The measurements are performed at $1 \mathrm{MHz}$. Numerical calculations with fabricated dimensions are in very good agreement with the experimental data.

In Figure 6, $\mathrm{C}-\mathrm{V}$ characteristics of various other MEMS varactors are shown. These devices have different number of comb-pairs $(N)$ and different spring designs $(w$ and $L$ ). As can be seen from this figure, depending on the tuning requirements, a maximum tuning voltage as low as $1.4 \mathrm{~V}$ can be used where a tuning between $0.9 \mathrm{pF}$ and $1.6 \mathrm{pF}$ is possible. It is worth mentioning here that there is a clear trade-off between the tuning voltage and electrical quality factor of an in-plane MEMS capacitor.

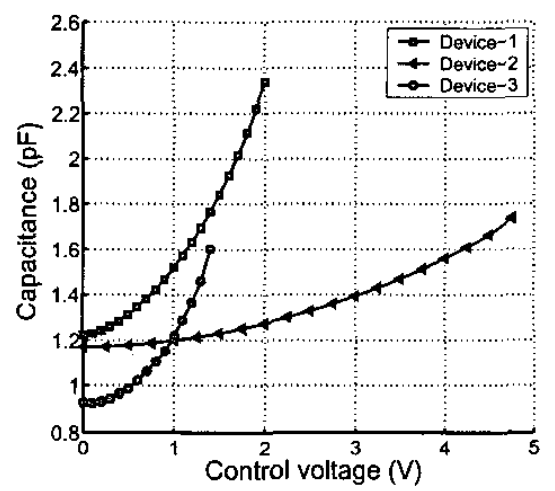

Figure 6. Capacitance-voltage tuning characteristic of the comb-driven tunable capacitors with different static capacitance (different number of combs) and different spring designs, measured at $1 \mathrm{MHz}$.

The dynamic behavior of the mechanical system was characterized at atmospheric pressure to extract the settling time using the C-t mode of the HP-4280A C-V plotter. An external pulse biasing is used to allow fast measurements with $10 \mu \mathrm{s}$ intervals. The transient behavior was measured by employing a pulse voltage excitation of 
$1.2 \mathrm{~V}$. The measurement was executed after the falling edge of the pulse to probe the recovery of the capacitor in time. As can be seen from Figure 7, the capacitor settles to the final value in about $200 \mu \mathrm{s}$, with the desired over-damped response. The reason for the noise in the capacitance after $0.2 \mathrm{~ms}$ is the limited resolution of the HP-4280A in C-t mode. Contrary to previously reported in-plane devices $[1,3]$, as a result of the over-damped transient response in air, the present device can safely be operated in ambient air without demanding a special package for the modification of medium viscosity or pressure.
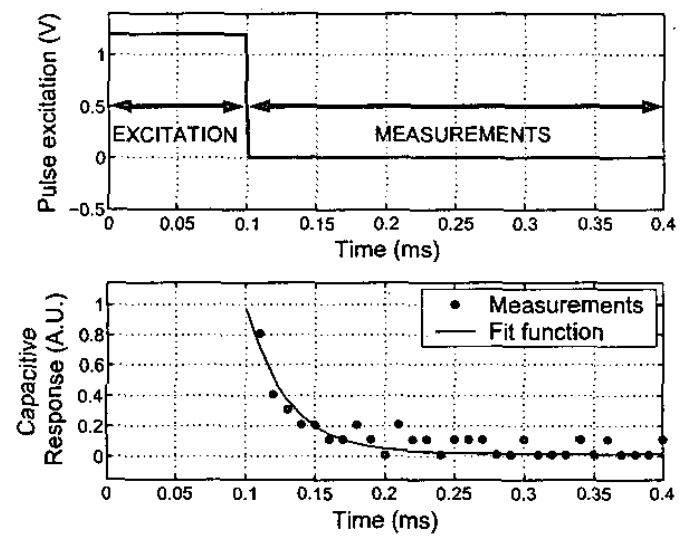

Figure 7. Transient response of the tunable capacitor. Measurements were performed after the falling edge of the excitation pulse.

The microwave performance of the device was characterized through two-port scattering parameters from which the self-resonance behavior and electrical quality factor can be extracted. The measurement setup consists of a Cascade probe station and ground-signal-ground configured probes with a standard pitch of $150 \mu \mathrm{m}$, connecting the device and the HP-8515A $s$-parameter test set through a micro-strip balanced coaxial cable. The HP8510 network analyzer was then used for measurements at microwave frequencies. Having calibrated the probes for open circuit, short-circuit and $50 \Omega$ load, the twoport $s$-parameters were measured in a frequency range of $45 \mathrm{MHz}$ to $10 \mathrm{GHz}$.

Figure 8 shows the $s_{11}$ parameter in a Smith chart, calculated from measurements from $45 \mathrm{MHz}$ to $10 \mathrm{GHz}$. From the measured $s_{11}$, the input impedance $\left(z_{11}\right)$ can be calculated by using $z_{11}=Z_{0} \frac{1+s_{11}}{1-s_{11}}$, having $Z_{0}=50 \Omega$ as the characteristic impedance. With a measured device capacitance of $1 \mathrm{pF}$; the real part of the $z_{11}$ parameter at $1 \mathrm{GHz}$ corresponds to a quality factor of 100 at $100 \mathrm{MHz}$ and roughly 10 at $1 \mathrm{GHz}$. The device exhibits a selfresonance at $4.08 \mathrm{GHz}$.

\section{Conclusion}

A high-aspect ratio tunable capacitor, that combines low-voltage actuation and wide tuning range, was suc-

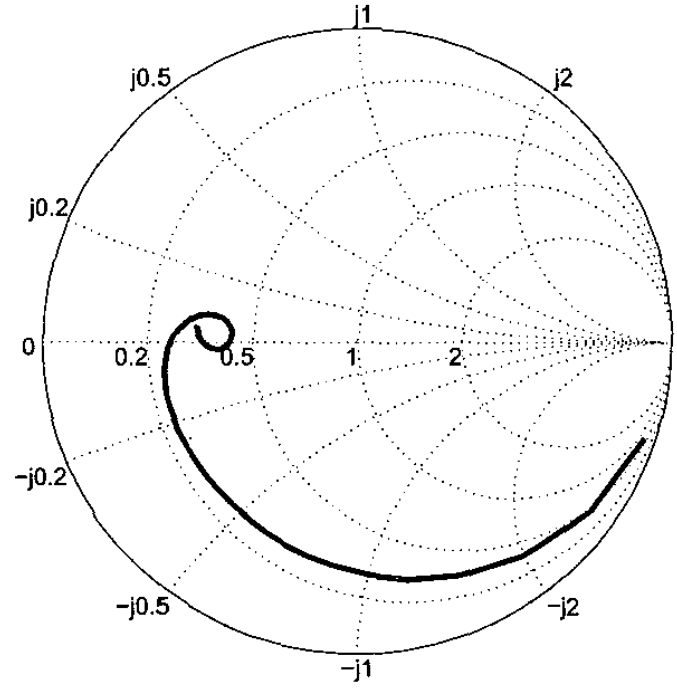

Figure 8. Reflection coefficient $\left(s_{11}\right)$ of the MEMS tunable capacitor measured from $45 \mathrm{MHz}$ to $10 \mathrm{GHz}$. (The measured two-port parameters were used to calculate one-port $s_{11}$ at the first port with shorted second port).

cessfully fabricated in a simple microfabrication process. An air gap aspect ratio of 1:20 was obtained with vertical sidewalls. Performance characterizations have proved that the present device can be utilized to implement VHF and UHF tunable filters and impedance matching networks with electrical quality factors well above 100 and the capacitance tuning ratios of $2: 1$ to $1.5: 1$. The present device family is compatible with the state of the art supply voltage range of integrated circuit technologies. The device does not to require any additional treatment for a desired, fast transient response. High frequency measurements revealed a self-resonance of $4.08 \mathrm{GHz}$, which safely falls at least one order of magnitude above the intended frequency band.

[1] R.L. Borwick III et al. "A high-Q, large tuning range, tunable capacitor for RF applications" IEEE Int. MEMS Conf. 2002, pp. 669-672 (2002).

[2] A. Dec and K. Suyama "Micromachined electromechanically tunable capacitors and their applications to RF IC's" IEEE Trans. on Microwave Theory and Techniques, 46, pp. 2587-2596 (1998).

[3] R.L. Borwick III et al., "A high-Q, large tuning range, MEMS capacitor for RF filter systems" Sens. and Act. A, 103, pp. 33-41 (2003).

[4] J.I. Seeger and S.B. Crary "Stabilization of electrostatically actuated mechanical device" Transducers 97, pp. 1133-1136, Chicago (1997).

[5] H. Nieminen et al., "Microelectromechanical capacitor with wide tuning range" IEE Electonics Letters, 37, pp. 1451-1452 (2001). 